\title{
Does early surgery improve outcomes for periprosthetic fractures of the hip and knee? A systematic review and meta-analysis
}

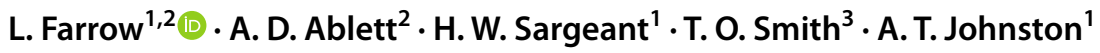

Received: 13 July 2020 / Accepted: 19 December 2020 / Published online: 8 February 2021

(c) The Author(s) 2021

\begin{abstract}
Introduction Previous evidence has established that early surgery is beneficial to improve outcomes for individuals with native hip fractures in the elderly population. Patients who sustain a periprosthetic fracture have been demonstrated to have similar demographics and outcomes as those with native fractures around the hip and knee. We therefore set out to determine if there is a similar difference in perioperative outcomes between early and delayed surgery for periprosthetic fractures of the hip and knee through a systematic review and meta-analysis.

Methods Literature search outputs were screened for studies meeting the inclusion criteria. The groups of early surgery and delayed surgery were defined by study authors. The primary outcome measure was 30 day mortality. Where there was sufficient study homogeneity, a random-effects meta-analysis was performed. Individual study risk of bias was assessed using the ROBINS-I criteria, with the GRADE criteria used for independent outcome evaluation. The review protocol was registered on PROSPERO prior to commencement (Registration number CRD42019149360).

Results The inclusion criteria was met in 11 studies $(n=3006)$. Mean time to surgery from admission for reporting studies was $64 \mathrm{~h} .59 .6 \%$ patients underwent early surgery as defined by the study authors. We identified a significantly lower risk of 30 day mortality for those with early surgery versus delayed surgery (RR $0.21 ; 95 \%$ CI $0.05,0.90 ; p=0.04, n=2022$ ). There were also significantly better outcomes for early versus delayed surgery regarding: medical complications, length of stay, transfusion risk, and reoperation. The quality of evidence for all the individual outcomes was low or very low.

Conclusions There is evidence that delaying surgery in those with periprosthetic fractures of the hip and knee has a deleterious impact on mortality and other important patient outcomes. There are, however, notable limitations to the existing available literature, with further appropriately designed large-scale studies required to confirm these findings.
\end{abstract}

Keywords Trauma $\cdot$ Orthopaedics $\cdot$ Periprosthetic $\cdot$ Fracture $\cdot$ Hip $\cdot$ Knee $\cdot$ Arthroplasty $\cdot$ Delay $\cdot$ Time to surgery $\cdot$ Systematic review

\section{Introduction}

Periprosthetic fractures around the hip and knee are an emerging problem across the orthopaedic community. Rates are expected to rise significantly in the future associated with dramatic increases in the amount of primary and revision arthroplasties performed, as well as an ageing population [22].

L. Farrow

luke.farrow@abdn.ac.uk

The patient cohort is similar to those who sustain a

1 Aberdeen Royal Infirmary, Foresterhill, Aberdeen AB25 2ZG, UK

2 Institute of Medical Sciences, University of Aberdeen, Foresterhill, Aberdeen AB25 2ZD, UK

3 Nuffield Department of Rheumatology, Orthopaedics and Musculoskeletal Sciences, Botnar Research Centre, University of Oxford, Windmill Road, Oxford OX3 7LD, UK fragility fracture of the hip or distal femur $[4,20]$, where there has been previous interest in time to surgery. There is now strong evidence for increased patient complications, including a higher mortality rate, when surgery is delayed in this setting [20, 25, 27, 28]. Many hip fracture guidelines now incorporate recommendations regarding minimal delay to surgery, typically within $36-48 \mathrm{~h}$ of 
admission [2, 23]. Current UK national guidance suggests that all frail patients requiring surgery should have this performed within $36 \mathrm{~h}$, which includes those with fragility fractures of the distal femur [5].

Given the similar reported mortality and morbidity for periprosthetic hip and knee fractures compared to native fractures $[4,20]$, it is perhaps surprising that periprosthetic fractures appear to often be associated with a significant delay from injury to theatre $[12,14,16]$. This is possibly related to difficulties regarding service provision for such fractures, where particular surgical expertise and specific implants are required. These challenges could, however, be overcome with appropriate planning and recognition if a clinical demand for prompt surgery was recognised. This has previously been seen in hip fracture surgery where national registries have demonstrated significant reductions in the time to theatre for the majority of patients $[23,29]$.

This systematic review and meta-analysis aimed to determine if delays to surgery for patients suffering periprosthetic fractures around hip or knee implants were associated with complication rates.

\section{Materials and methods}

The study was reported in accordance with the PRISMA (Preferred Reporting Items for Systematic Reviews and Meta-Analyses) guidance [19]. The review protocol was registered on the international prospective register of systematic reviews (PROSPERO) prior to commencement (Registration number CRD42019149360). Hip and knee periprosthetic fractures were included together due to the relative scarcity of cases, with both conditions occurring in the same patient cohort, and having similar reported outcomes for native hip and distal femoral fractures [20, 25].

\section{Search strategy}

Medline, EMBASE, and the Cochrane Central Registry of Controlled Trials were investigated for relevant outputs using the OvidSP search platform. Additional searches using the Google search engine, the WHO clinical trial registry, clinicaltrials.gov, and the OpenGrey database were also performed to ensure that all potentially eligible materials were identified; reference lists of relevant articles were also screened. All electronic searches were undertaken from database inception to October 2019. The only limit placed on the search strategy was articles in the English language. The full electronic search strategy is presented in "Appendix 1".

\section{Eligibility criteria}

Included studies were non-review research articles (clinical trials, case series, and other observational study methods) reporting on clinical outcomes relating to periprosthetic fractures around the hip and knee. This had to include analysis regarding time to surgery. The intervention and control groups were early surgery and delayed surgery (as defined by the study authors e.g., $<24 \mathrm{~h}$ and $\geq 24 \mathrm{~h}$ ) respectively. Only studies that considered time to surgery as an independent categorical variable were included. Studies including periprosthetic fractures in areas other than the hip and knee, periprosthetic patella fractures, non-operative management, open fractures, and neurovascular injury were excluded. No restrictions were placed on the level of evidence presented through individual studies or the study design.

\section{Study identification}

Studies were identified from screened abstracts of potentially appropriate studies which met the eligibility criteria by two independent reviewers (LF and AA). Selected studies then proceeded to full-text assessment where a final decision on suitability was made. Any discrepancy regarding study eligibility was decided by reviewer discussion.

\section{Data extraction}

Two assessors (AA and HS) independently extracted relevant data from each study using standardised data forms for both patient demographics and outcomes. Demographic data included: study year, study design, number participants, gender, age, American Society of Anaesthesiologists physical status classification (ASA), Charlson Co-morbidity Index, osteoporosis, dementia, time from initial surgery to fracture, type of periprosthetic fracture (hip/knee/both), Vancouver/ Unified classification system, time to surgery, type of surgery (revision/osteosynthesis/both), anaesthetic type, duration of surgery, and intervention/control group (if comparing time to surgery). In the instance of missing data, attempts were made to contact study authors. Outcome data fields are detailed below.

\section{Outcome measures}

The primary outcome measure was 30 day mortality. Secondary outcome measures included: 1-year mortality, length of stay, transfusion, all-cause medical complications (respiratory, e.g., pneumonia, cardiovascular, e.g., myocardial infarction/stroke, renal, e.g., urinary tract infection and acute 
kidney injury, and sepsis), surgical site infection, and allcause reoperation (infection, dislocation, and implant failure/ fracture).

\section{Quality assessment}

Risk of bias in observational studies was assessed using The Risk of Bias in Non-randomised Studies-of Interventions (ROBINS-I) tool [26]. This was performed independently by two reviewers (LF and AA). Bias assessment was also performed for each individual outcome using the Grading Quality of Evidence and Strength of Recommendations (GRADE) criteria [3].

\section{Statistical analysis}

Where there were a minimum of two studies categorically assessing time to surgery as an independent variable, with sufficient homogeneity regarding the study design, intervention, and population, a meta-analysis was undertaken using Revman 5.3 [1]. For cases where the included studies did not meet the criteria for meta-analysis, a narrative review was performed. Where meta-analysis was performed, a randomeffects model was utilised. Standard mean difference (SMD) was calculated for continuous outcomes, with relative risk (RR) for dichotomous variables. In all analyses $p<0.05$ denoted statistical significance. Analyses are presented with $95 \%$ confidence intervals.

\section{Results}

The search results are summarised in Fig. 1. A total of 144 studies were identified, with 13 undergoing full-text review. 11 articles $[4,6-8,10,12,13,15,16,18,24]$ were determined to be eligible for study inclusion.

\section{Study characteristics}

A summary of included studies is shown in Table 1. A total of 3006 participants were included. Mean time to fracture from index procedure was 7.6 years for reporting studies. Mean time to surgery from admission for reporting studies was $64 \mathrm{~h}$. In the two studies that reported dichotomous time to surgery at $24 \mathrm{~h}[6,7], 46.9 \%$ (402/857) and $77.9 \%$ (377/484) participants were found to have received surgery within $24 \mathrm{~h}$ respectively. Three studies reported dichotomous time to surgery at $48 \mathrm{~h}$ [8, 18, 24], with $31.1 \%(56 / 180), 60.1 \%$ (409/681), and $216 / 263(82 \%)$ of individuals receiving surgery within this time frame. All 11 articles included in the study were of a retrospective cohort design. Individual risk of bias

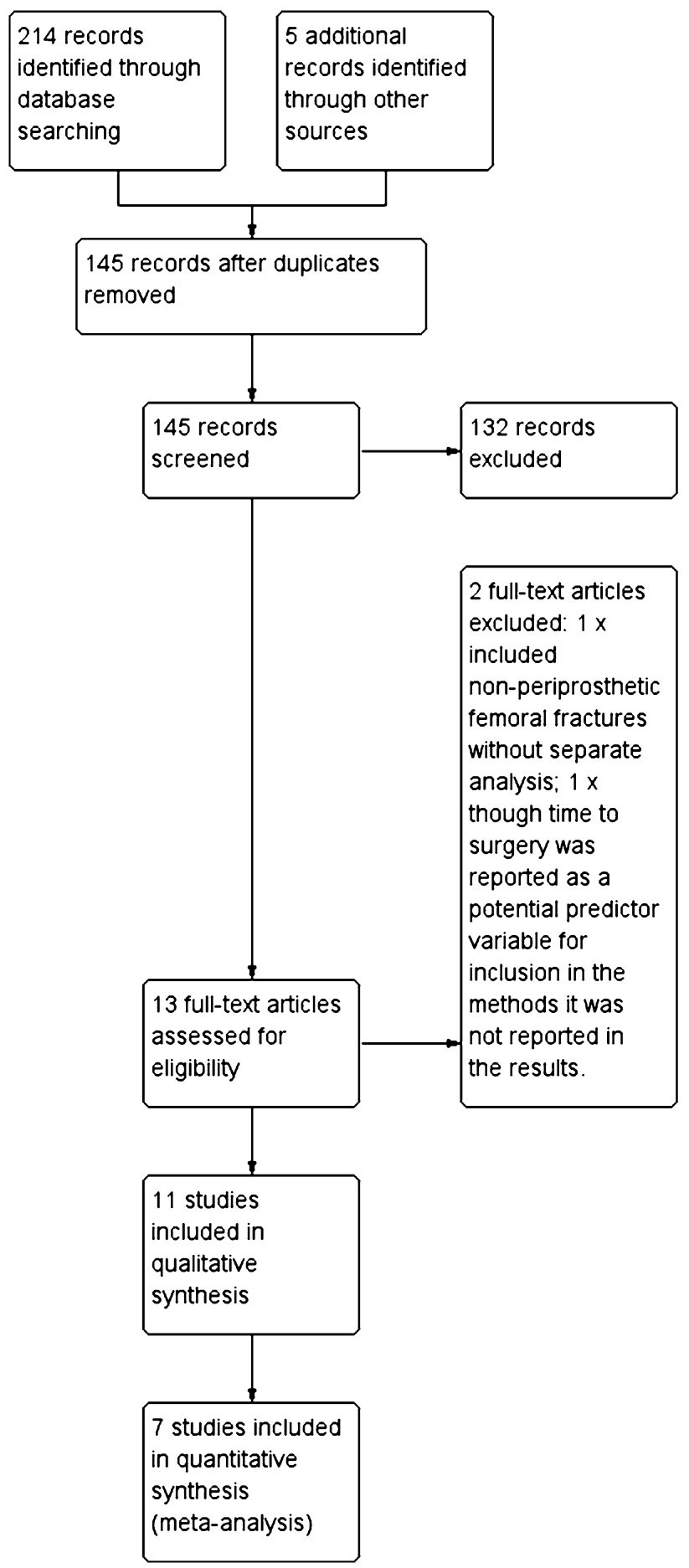

Fig. 1 Flow diagram of study selection process

assessment was performed for each study according to the ROBINS-I tool, with results shown in supplementary Table 1. Results for the GRADE analysis are reported for individual outcomes, with a summary contained within supplementary table 2 . 


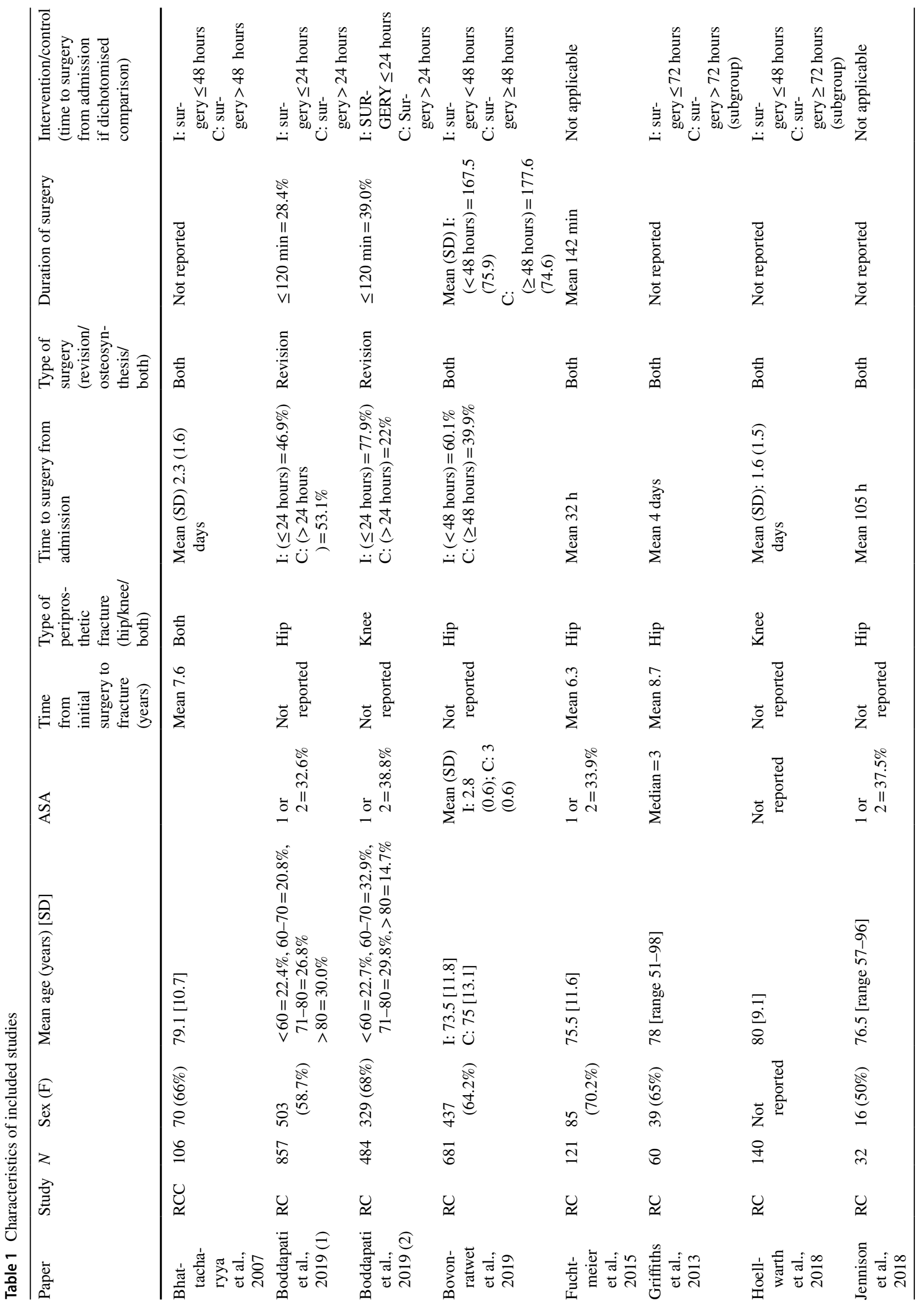




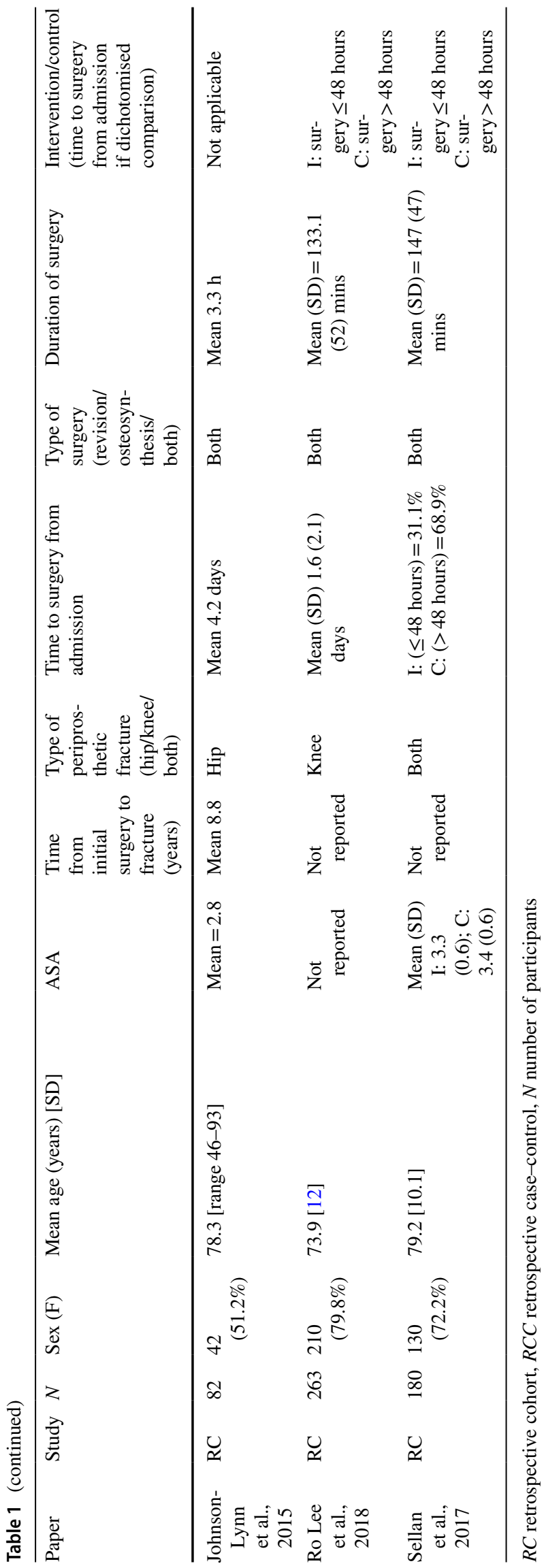

Primary outcome: 30 day mortality

Three studies reported 30 day mortality with a dichotomised delay to surgery [6-8]. On meta-analysis, there was a significantly lower risk of 30 day mortality for those with early surgery versus delayed surgery (RR $0.21 ; 95 \%$ CI $0.05,0.90$; $p=0.04, n=2022$-Fig. 2). All other studies did not report on 30 day mortality. On GRADE assessment, the quality of evidence was found to be very low due to observational design, serious imprecision, and serious inconsistency.

\section{1-Year mortality}

Three studies reported 1-year mortality with a dichotomised delay to surgery $[4,13,24]$. On meta-analysis, there was a trend towards a lower mortality at 1 year for early versus delayed surgery, but no statistically significant difference (RR 0.61; 95\% CI 0.36, 1.03; $p=0.06, n=407$-supplementary Fig. 1). In addition Fuchtmeier et al. 2015 reported on 1 year mortality and found no significant difference in mean delay to surgery for those with no mortality $(21.1 \mathrm{~h})$ and those with mortality (27.2 h); $p=0.60$. Jennison and Yarlagadda [15] also found no significant difference in 1 year mortality in mean delay to surgery for those with no mortality (109h) versus those with mortality (104h); $p=0.88$. On GRADE assessment, the level of evidence was found to be very low due to observational design, a serious risk of bias, serious imprecision, and serious inconsistency.

\section{Length of stay}

Two studies reported length of stay with a dichotomised delay to surgery as greater than or less than $24 \mathrm{~h}[6,7]$. On meta-analysis, there was a significantly shorter length of stay for those operated on within $24 \mathrm{~h}$, versus those who waited longer (SMD -1.03 days; 95\% CI $-1.88,-0.19 ; p=0.02$, $n=1341$ - supplementary Fig. 2). Conversely, JohnsonLynn et al. [16] found that there was no significant positive correlation between delay to surgery and overall length of stay (Pearson correlation coefficient $-0.1191, n=82$ ). On GRADE assessment, the level of evidence was found to be very low due to observational design, serious imprecision, and serious inconsistency.

\section{Transfusion}

Four studies reported on transfusion rate with a dichotomised delay to surgery $[4,6,7,18]$. On meta-analysis, there was a significantly lower transfusion rate for those with an early operation, compared to those with a delay to surgery (RR $0.51 ; 95 \%$ CI $0.31,0.82 ; p=<0.001$, 


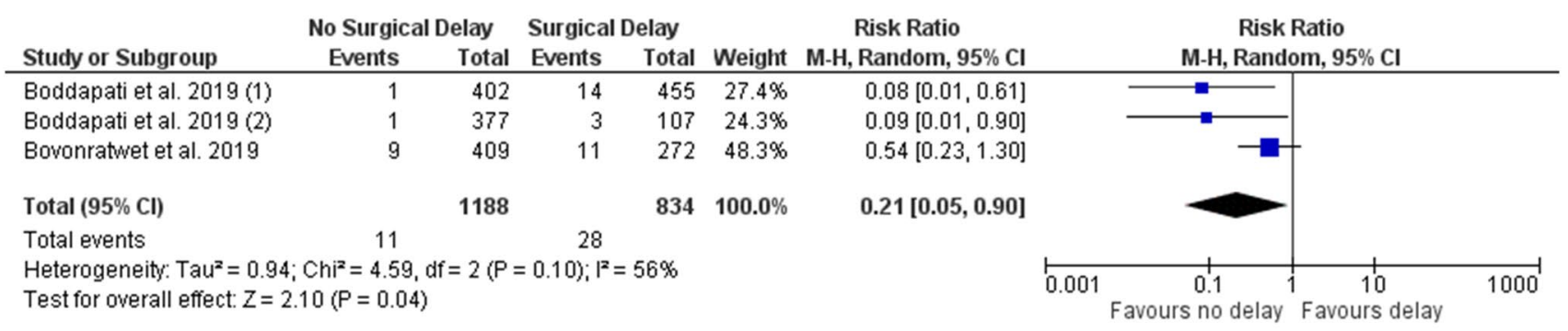

Fig. 2 Forest plot of delayed versus early surgery for the outcome of 30 day mortality

$n=2285-$ Supplementary Fig. 3). On GRADE assessment, the level of evidence was found to be very low due to observational design and serious inconsistency.

\section{Medical complications (all cause)}

Five studies reported on medical complications with a dichotomised delay to surgery $[6-8,18,24]$. On meta-analysis, there was a significantly lower medical complication rate for those with early surgery versus delayed surgery $(\mathrm{RR}$ 0.58 ; 95\% CI 0.42, 0.82; $p=0.002, n=2465$-Supplementary Fig. 4). Griffiths et al. 2013 [12] also reported on the influence of surgical delay on post-operative complications (although this included both medical and surgical complications). They found that patients with no complications had a significantly shorter surgical delay (2.82 days) compared to those with complications ( 5.41 days) $(p=0.02)$. JohnsonLynn et al. [16], on the other hand, did not find a correlation between the number of post-operative complications and an increasing delay to surgery (Pearson correlation coefficient $-0.0444, n=82$ ). On GRADE assessment, the level of evidence was found to be very low due to observational design and serious inconsistency.

\section{Surgical site infection}

Five studies reported on surgical site infection with time to surgery as an independent categorical variable $[6-8,18$, 24]. On meta-analysis, there was a trend towards a lower surgical site infection rate with a shorter delay to surgery, but this was not statistically significant (RR $0.61 ; 95 \%$ CI 0.36, 1.03; $p=0.06, n=2465$-Supplementary Fig. 5). On GRADE assessment, the level of evidence was found to be low due to observational design.

\section{Reoperation (all cause)}

Three studies reported on all-cause reoperation with a dichotomised delay to surgery [6-8]. On meta-analysis, there was a significantly lower reoperation rate for those with early surgery compared to delayed surgery (RR $0.63,95 \%$ CI 0.45 ,
0.89; $p=<0.001, n=2022$-Supplementary Fig. 6). There were insufficient data available to split reoperation by cause (e.g., infection, dislocation, implant failure, or fracture). On GRADE assessment, the level of evidence was found to be low due to observational design.

\section{Discussion}

This systematic review and meta-analysis found that there is weak evidence, as defined by the GRADE criteria, to suggest a delay to surgery for patients with a periprosthetic fracture of either the hip or knee is associated with worse patient outcomes. This included increased 30 day mortality, greater likelihood of medical complications, longer length of stay, greater risk of transfusion, and reoperation. There were also trends identified towards a greater risk of 1 year mortality and surgical site infection.

These findings are similar to those previously identified in native hip [25] and distal femoral [20] fractures, where prompt surgery has been associated with improved outcomes. As demonstrated by the low-quality evidence identified during this study, the links in the periprosthetic fracture setting are less concrete, with a clear need for further research to confirm our findings. Given the study design limitations of the current evidence, it is difficult to discern whether the associations observed in this work are due to causal effect from delay to surgery, or patient selection bias. Nevertheless, taking into account the similarities in the hip fracture and periprosthetic fracture cohorts [4], clinical reasoning would support the hypothesis that the impact of a delay to surgery would be similar for both groups. Current UK national guidance suggests that all frail patients should be managed with urgent surgery to quickly restore mobility and reduce associated complications [5].

One of the potential issues with managing time to surgery for periprosthetic fractures is that there are currently significant service provision limitations that would have to be addressed prior to implementing improvements in surgical delay. This is particularly true for revision surgery, where specialist hip surgeon knowledge and skills are often required, and may 
not be immediately available. A number of studies included in this analysis confirmed that patients undergoing revision surgery had longer waiting times compared to those treated with fixation alone $[8,13,15]$. Given that revision surgery for Vancouver B2 fractures has already been associated with mortality and morbidity benefits compared to fixation alone [17], these results may actually be even more pronounced when taking into account the impact of surgical delay. Examination of different models of service provision, such as a specific arthroplasty on-call service providing reliable access to surgical expertise, would undoubtedly be beneficial in the ultimate implementation of expedited surgery for these patients.

When considering future studies within this area, one of the issues demonstrated throughout our analysis is a lack of consistent reporting and outcomes between studies. Despite 11 articles included in the systematic review, only 7 were suitable for inclusion within a meta-analysis. In addition, many of these studies utilised different methodologies and reported on different outcomes. Development of a consensus core dataset such as that suggested by Khan et al. [17] in their systematic review would be of benefit to ensure consistent reporting across studies, and aid in conducting meaningful comparisons.

Establishment of national or international periprosthetic fracture registries would also be of benefit in further understanding the impact of time to surgery for these injuries given their relatively low incidence. Such registries would allow for the completion of large-scale cohort studies, which would be particularly useful given the difficulties in patient recruitment and ethical concerns for performing randomised-controlled trials in this setting. Techniques such as propensity matching for known confounders would help mitigate the potential bias from patient selection; particularly as a number of other factors potentially influential in periprosthetic fracture outcomes have been previously described $[11,17,21]$. The use of large-scale registry data should also allow for the separation of periprosthetic knee and periprosthetic hip fracture patients into individual cohorts, as a previous report has suggested the possibility of difference in demographics and outcome between the two cohorts [9]. Using the results from our analysis, we have calculated an estimated sample size for such a study examining dichotomised time to surgery and 30 day mortality would require 1170 participants to appropriately investigate this primary outcome (https://www.clincalc.com/stats/sampl esize.aspx). None of the current included studies met this requirement (range 32-857 participants).

\section{Conclusion}

Current evidence suggests that delaying hip and knee periprosthetic fracture surgery is associated with higher patient morbidity and mortality. Given the low quality of this evidence, further large-scale studies, with appropriate adjustment for confounding bias, are required to confirm these findings. Understanding the service provision barriers that prevent early surgery for these patients would help to design interventions to reduce delay and consequently improve key patient healthcare outcomes.

Supplementary Information The online version contains supplementary material available at https://doi.org/10.1007/s00402-020-03739-2.

Author contributions LF: developed research idea, designed study, performed data extraction and analysis, performed interpretation of results, and drafted and revised manuscript. ADA: performed data extraction and analysis, and drafted and revised manuscript. HWS: performed data extraction, and drafted and revised manuscript. TOS: performed interpretation of results, and drafted and revised manuscript. ATJ: performed interpretation of results, and drafted and revised manuscript.

Funding None.

\section{Compliance with ethical standards}

Conflict of interest The authors declare that they have no conflict of interest.

Consent for participation/publication Not required.

Open Access This article is licensed under a Creative Commons Attribution 4.0 International License, which permits use, sharing, adaptation, distribution and reproduction in any medium or format, as long as you give appropriate credit to the original author(s) and the source, provide a link to the Creative Commons licence, and indicate if changes were made. The images or other third party material in this article are included in the article's Creative Commons licence, unless indicated otherwise in a credit line to the material. If material is not included in the article's Creative Commons licence and your intended use is not permitted by statutory regulation or exceeds the permitted use, you will need to obtain permission directly from the copyright holder. To view a copy of this licence, visit http://creativecommons.org/licenses/by/4.0/.

\section{References}

1. Review Manager (RevMan) [Computer program]. Version 5.3 (2014) The Nordic Cochrane Centre, The Cochrane Collaboration, Copenhagen

2. PHS (2018) Scottish standards of care for hip fracture patients. https://www.shfa.scot.nhs.uk/_docs/2018/Scottish-standards-ofcare-for-hip-fracture-patients2018.pdf

3. Atkins D, Best D, Briss PA et al (2004) Grading quality of evidence and strength of recommendations. BMJ 328(7454):1490

4. Bhattacharyya T, Chang D, Meigs JB, Estok DM 2nd, Malchau H (2007) Mortality after periprosthetic fracture of the femur. J Bone Jt Surg Am 89(12):2658-2662

5. BOA, BGS, NHFD, FFN (2019) BOAST: the care of the older or frail orthopaedic trauma patient. https://www.bgs.org.uk/ sites/default/files/content/attachment/2019-06-03/BOA\%20FFN \%20BGS\%20BOAST_0.pdf

6. Boddapati V, Grosso MJ, Sarpong NO, Geller JA, Cooper HJ, Shah RP (2019) Early morbidity but not mortality increases with surgery delayed greater than 24 hours in patients with a 
periprosthetic fracture of the hip. J Arthroplast 34(11):2789 e2781-2792 e2781

7. Boddapati V, Held MB, Lee NJ, Geller JA, Cooper HJ, Shah RP (2019) Is the time to revision surgery after peri-prosthetic fracture of the knee associated with increased rates of post-operative complications? Arthroplast Today 5(3):348-351

8. Bovonratwet P, Fu MC, Adrados M, Ondeck NT, Su EP, Grauer JN (2019) Unlike native hip fractures, delay to periprosthetic hip fracture stabilization does not significantly affect most short-term perioperative outcomes. J Arthroplast 34(3):564-569

9. Eschbach D, Buecking B, Kivioja $\mathrm{H}$ et al (2018) One year after proximal or distal periprosthetic fracture of the femur -two conditions with divergent outcomes? Injury 49(6):1176-1182

10. Fuchtmeier B, Galler M, Muller F (2015) Mid-term results of 121 periprosthetic femoral fractures: increased failure and mortality within but not after one postoperative year. J Arthroplast 30(4):669-674

11. Gibbs VN, McCulloch RA, Dhiman P et al (2020) Modifiable risk factors for mortality in revision total hip arthroplasty for periprosthetic fracture. Bone Jt J 102-B(5):580-585

12. Griffiths EJ, Cash DJ, Kalra S, Hopgood PJ (2013) Time to surgery and 30-day morbidity and mortality of periprosthetic hip fractures. Injury 44(12):1949-1952

13. Hoellwarth JS, Fourman MS, Crossett L et al (2018) Equivalent mortality and complication rates following periprosthetic distal femur fractures managed with either lateral locked plating or a distal femoral replacement. Injury 49(2):392-397

14. Jennison T, Yarlagadda R (2018) Mortality in patients sustaining a periprosthetic fracture following a hemiarthroplasty. J Orthop 15(3):798-801

15. Jennison T, Yarlagadda R (2018) Mortality in patients sustaining a periprosthetic fracture following a previous extracapsular hip fracture fixation. Injury 49(3):702-704

16. Johnson-Lynn S, Ngu A, Holland J, Carluke I, Fearon P (2016) The effect of delay to surgery on morbidity, mortality and length of stay following periprosthetic fracture around the hip. Injury 47(3):725-727

17. Khan T, Grindlay D, Ollivere BJ, Scammell BE, Manktelow AR, Pearson RG (2017) A systematic review of Vancouver B2 and B3 periprosthetic femoral fractures. Bone Jt J 99-B(4 Supple B): 17-25

18. Lee SR, Shrestha K, Staggers J, Li P, Naranje SM, Shah A (2018) Impact of time to surgery from injury on postoperative infection and deep vein thrombosis in periprosthetic knee fractures. Chin J Traumatol 21(6):329-332

19. Moher D, Liberati A, Tetzlaff J, Altman DG, Group P (2009) Preferred reporting items for systematic reviews and meta-analyses: the PRISMA statement. BMJ 339:b2535

20. Myers P, Laboe P, Johnson KJ et al (2018) Patient mortality in geriatric distal femur fractures. J Orthop Trauma 32(3):111-115

21. Orfanos G, Lim J, Youssef B (2019) Evaluating risk factors following surgery for periprosthetic fractures around hip and knee arthroplasties. Arch Orthop Trauma Surg 139(4):475-482

22. Pivec R, Issa K, Kapadia BH et al (2015) Incidence and future projections of periprosthetic femoral fracture following primary total hip arthroplasty: an analysis of international registry data. J Long Term Eff Med Implants 25(4):269-275

23. RCP (2016) National hip fracture database annual report 2016. http://web1.crownaudit.org/Report2016/NHFD2016Report.pdf

24. Sellan ME, Lanting BA, Schemitsch EH, MacDonald SJ, Vasarhelyi EM, Howard JL (2018) Does time to surgery affect outcomes for periprosthetic femur fractures? J Arthroplast 33(3):878-881

25. Shiga T, Wajima Z, Ohe Y (2008) Is operative delay associated with increased mortality of hip fracture patients? Systematic review, meta-analysis, and meta-regression. Can J Anaesth 55(3): 146-154

26. Sterne JA, Hernan MA, Reeves BC et al (2016) ROBINS-I: a tool for assessing risk of bias in non-randomised studies of interventions. BMJ 355:i4919

27. Taylor M, Hopman W, Yach J (2016) Length of stay, wait time to surgery and 30-day mortality for patients with hip fractures after the opening of a dedicated orthopedic weekend trauma room. Can J Surg 59(5):337-341

28. Weller I, Wai EK, Jaglal S, Kreder HJ (2005) The effect of hospital type and surgical delay on mortality after surgery for hip fracture. J Bone Jt Surg Br 87(3):361-366

29. Zanardi DM, Parpinelli MA, Haddad SM et al (2019) Adverse perinatal outcomes are associated with severe maternal morbidity and mortality: evidence from a national multicentre cross-sectional study. Arch Gynecol Obstet 299(3):645-654

Publisher's Note Springer Nature remains neutral with regard to jurisdictional claims in published maps and institutional affiliations. 